\title{
A single-arm pilot study of metformin in patients with autosomal dominant polycystic kidney disease
}

Bogdan Marian Sorohan ${ }^{1,2}$, Gener Ismail ${ }^{1,2^{*}}$, Andreea Andronesi ${ }^{1,2}$, Georgia Micu², Bogdan Obrișcă ${ }^{1,2}$, Roxana Jurubiță ${ }^{1,2}$, loanel Sinescu ${ }^{1,3}$ and Cătălin Baston ${ }^{1,3}$

\begin{abstract}
Background: Metformin has shown promising results regarding cystogenesis inhibition in preclinical studies with autosomal dominant polycystic kidney disease (ADPKD) models. We designed a prospective, preliminary, single-arm study to evaluate the tolerability, safety and the effect of Metformin on kidney function and body mass index (BMI) in Romanian patients with ADPKD.

Methods: We enrolled 34 adult patients with ADPKD, chronic kidney disease (CKD) stages 1-5 not on dialysis and without diabetes mellitus. The primary endpoint was to assess the tolerability and safety of Metformin. The secondary endpoints evaluated changes in estimated glomerular filtration rate (eGFR), body mass index (BMI) and renal replacement therapy (RRT) necessity. Patients received an initial dose of Metformin of $500 \mathrm{mg} /$ day within the first month that was increased to $1000 \mathrm{mg} /$ day thereafter according to tolerability. Change in eGFR and BMI was expressed as mean difference with the corresponding 95\% confidence intervals and as a percentage. For the primary endpoint, we included all 34 enrolled patients. To assess the secondary endpoint, intention-to-treat (ITT) and per-protocol (PP) analysis was performed.

Results: Sixteen patients out of 34 completed the follow-up period at 24 months. Eighteen patients developed adverse events and $63.6 \%$ of these events were gastrointestinal related. Nausea was the most common adverse event (17.6\%). Two patients (5.8\%) permanently discontinued medication due to adverse events. We recorded no case of hypoglycemia, lactic acidosis or death. Mean eGFR changed by $-1.57 \mathrm{ml} / \mathrm{min} / 1.73 \mathrm{~m}^{2}$ (95\% $\mathrm{Cl}$ :-22.28 to 19.14 , $P=0.87)$ in IT and by $-4.57 \mathrm{ml} / \mathrm{min} / 1.73 \mathrm{~m}^{2}(95 \% \mathrm{Cl}:-28.03$ to $18.89, P=0.69)$ in PP population. Mean BMl change was $-1.10 \mathrm{~kg} / \mathrm{m}^{2}(95 \% \mathrm{Cl}:-3.22$ to $1.02, P=0.30)$ in IT population and $-0.80 \mathrm{~kg} / \mathrm{m}^{2}(95 \% \mathrm{Cl}:-3.27$ to $1.67, P=0.51)$ in PP analysis. Three patients (8.8\%) needed RRT.
\end{abstract}

Conclusions: Metformin was well tolerated, had a good safety profile even in ADPKD patients with advanced CKD and it was not associated with change in eGFR or BMI across the follow-up period.

Trial registration: The study was retrospectively registered on https://www.isrctn.com (number ISRCTN 93749377); date registered: 02/25/2019.

Keywords: Autosomal dominant polycystic kidney disease, Metformin, Glomerular filtration rate, Body mass index, Tolerability

\footnotetext{
* Correspondence: gener732000@yahoo.com

${ }^{1}$ Carol Davila University of Medicine and Pharmacy, Bucharest, Romania

${ }^{2}$ Nephrology Department, Fundeni Clinical Institute, Fundeni Street No. 258,

ZIP Code 022328, District No.2, Bucharest, Romania

Full list of author information is available at the end of the article
}

(c) The Author(s). 2019 Open Access This article is distributed under the terms of the Creative Commons Attribution 4.0 International License (http://creativecommons.org/licenses/by/4.0/), which permits unrestricted use, distribution, and reproduction in any medium, provided you give appropriate credit to the original author(s) and the source, provide a link to the Creative Commons license, and indicate if changes were made. The Creative Commons Public Domain Dedication waiver (http://creativecommons.org/publicdomain/zero/1.0/) applies to the data made available in this article, unless otherwise stated. 


\section{Background}

Autosomal Dominant Polycystic Kidney Disease (ADPKD) is the most common hereditary kidney disease, characterized by multiple, bilateral renal cysts leading to an increased kidney volume and progressive loss of kidney function. It is a systemic disorder that also affects the liver, pancreas, spleen, seminal vesicles, arachnoid membrane and endothelium [1]. Recent epidemiologic studies reported an average prevalence of ADPKD in Europe of 2.7: $10,000(95 \% \mathrm{CI}=0.73-4.67)$ and a point prevalence of 3.96:10,000 (95\% CI $=0.94-0.98)$ in countries from the European Union [2, 3]. According to the European Renal Association-European Dialysis and Transplant Association (ERA-EDTA) Registry, the estimated prevalence of ADPKD in Romania was 1.9:10,000 in 2012 [3]. PKD 1 and PKD 2 genes mutations are involved in the majority of cases, but recently new gene players were described, such as GANAB and DNAJB11 $[4,5]$. Polycystin 1 and 2 encoded by PKD genes are expressed mainly in tubular epithelial cells and work physiologically as an unit with well defined functional and structural roles [6]. In ADPKD, the abnormality of polycystin 1 and 2 proteins leads to primary cilia dysfunction, activation of several intracellular pathways, increased fluid secretion, cell-cell adhesion issues and hyperproliferation. Among the most important intracellular pathways involved were cyclic adenosine monophosphate (cAMP), mechanistic target of rapamycin (mTOR), mitogen-activated protein kinase/ extracellular signal-regulated kinase (MAPK/ERK), adenosine monophosphate-activated protein kinase (AMPK), just another kinase/signal transducer and activator of transcription (JAK/STAT) [7]. During the last decade, different therapeutic agents were used to counteract cyst and kidney dysfunction progression [8]. Metformin, the most common used biguanide for the treatment of type 2 diabetes mellitus, with a good safety profile, has shown promising results regarding cystogenesis inhibition in preclinical studies $[9,10]$. The proposed mechanism of action in ADPKD is by stimulating AMPK activation, which negatively regulates both the chloride channel cystic fibrosis transmembrane conductance regulator (CFTR) from the apical membrane and mTOR pathway. CFTR activity is directly inhibited and that of the mTOR pathway is indirectly inhibited through phosphorylation protein of tuberous sclerosis 2 (TSC2) and Raptor, leading to the blockage of two essential processes of cystogenesis, fluid secretion and proliferation [9]. Evolution of kidney function in patients with ADPKD is characterized by a high individual variability. Several environmental, genetic, demographic, clinical, biological and structural factors were associated with disease progression [11]. Among them, overweight and particularly, obesity were associated with greater kidney function decline and disease progression [12]. It has already been demonstrated that
Metformin promotes weight loss in overweight and obese patients [13]. Apart from the direct effect on cystogenesis, Metformin could have a beneficial additional effect in controlling the decline of renal function by producing a decrease in body mass index (BMI).

On the basis of these observations, we designed a prospective, preliminary, single-arm study to evaluate the tolerability, safety and the effect of Metformin on kidney function and BMI in Romanian patients with ADPKD.

\section{Methods \\ Study design}

METformin in ROmanian Patients with autosomal dominant polycystic kidney disease (METROP) was designed as a pilot, single center, interventional, single-arm study to investigate the safety, tolerability of Metformin and the change in kidney function and BMI under treatment, in Romanian adult patients with ADPKD.

\section{Inclusion and exclusion criteria}

Between April 2016 and December 2016, 34 patients were enrolled (Fig. 1). Inclusion criteria were: adult patients (age $\geq 18$ years), diagnosis of ADPKD based on unified ultrasonographic Pei-Ravine criteria [14], CKD stages between 1 and 5 not on dialysis. We ruled out: patients with diabetes mellitus, any patient with active infections, pregnant or breastfeeding patients, those with known contraindication or allergy to Metformin and those on renal replacement therapy (RRT).

\section{Endpoints}

The primary endpoint was to assess the tolerability and safety of Metformin in patients with ADPKD. The tolerability outcome included evaluation of the number and type of gastrointestinal and non-gastrointestinal symptoms. The safety outcome analyzed the presence of hypoglycemia, lactic acidosis, death and other adverse events. Also, the percentage of patients that permanently discontinued medication due to adverse events was studied.

The secondary endpoints evaluated the change in kidney function from baseline, number/percentage of patients that needed RRT and change in BMI from the baseline value after 12 and 24 months of treatment with Metformin.

\section{Metformin dosage}

Patients received an initial dose of Metformin of 500 $\mathrm{mg} /$ day within the first month, that was increased to $1000 \mathrm{mg} /$ day (500 mg twice daily), depending on tolerance and adverse events. Drug initiation and dose augmentation were made in the hospital. If patients declared that medication was well tolerated, with no severe gastrointestinal symptoms and if no hypoglycemia 


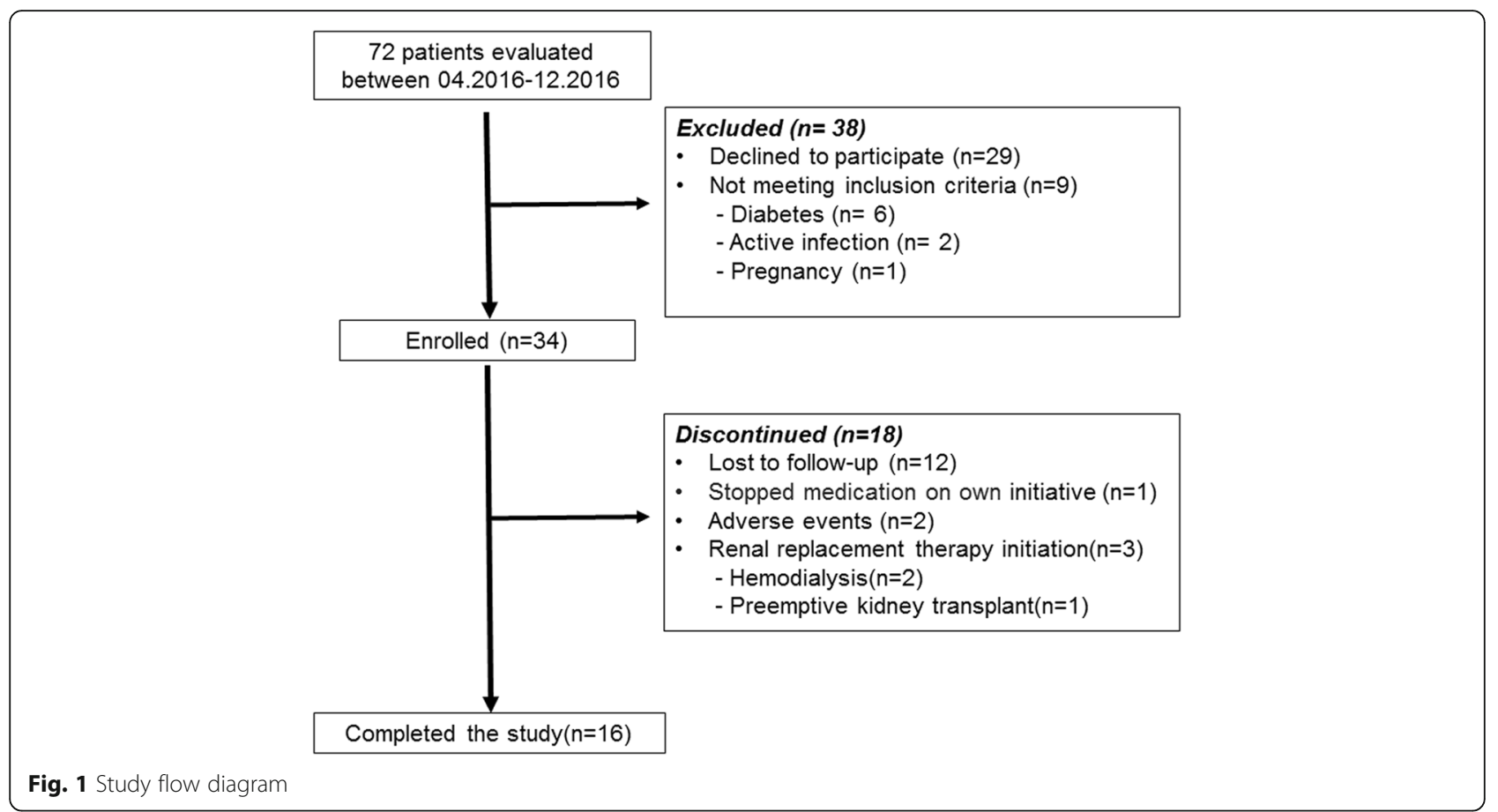

or lactic acidosis was found in the laboratory tests, the dose was increased to $1000 \mathrm{mg} /$ day after the first month. The dose of Metformin in patients with CKD stage 5 was limited to $500 \mathrm{mg} /$ day throughout the duration of the study.

\section{Study follow-up and data collection}

The study follow-up period was of 24 months. In the first year, visits were established at 1, 4 and 12 months, and after this period, at 18 and 24 months. At baseline, data regarding personal medical history, family history of ADPKD, demographic, smoking status and antihypertensive drugs were collected. Also, at baseline and at each study visit, patients were questioned about drug tolerability, underwent physical examination, including BMI assessment and laboratory tests were performed, including: glycemic and lipid profiles, liver tests, renal function tests, lactic acid levels, complete blood count and urinary tests (Fig. 2).

Safety, tolerability, kidney function and body mass index Gastrointestinal tolerability of Metformin was assessed by evaluating the occurrence rates of symptoms such as nausea, vomiting, diarrhea, bloating, abdominal pain and

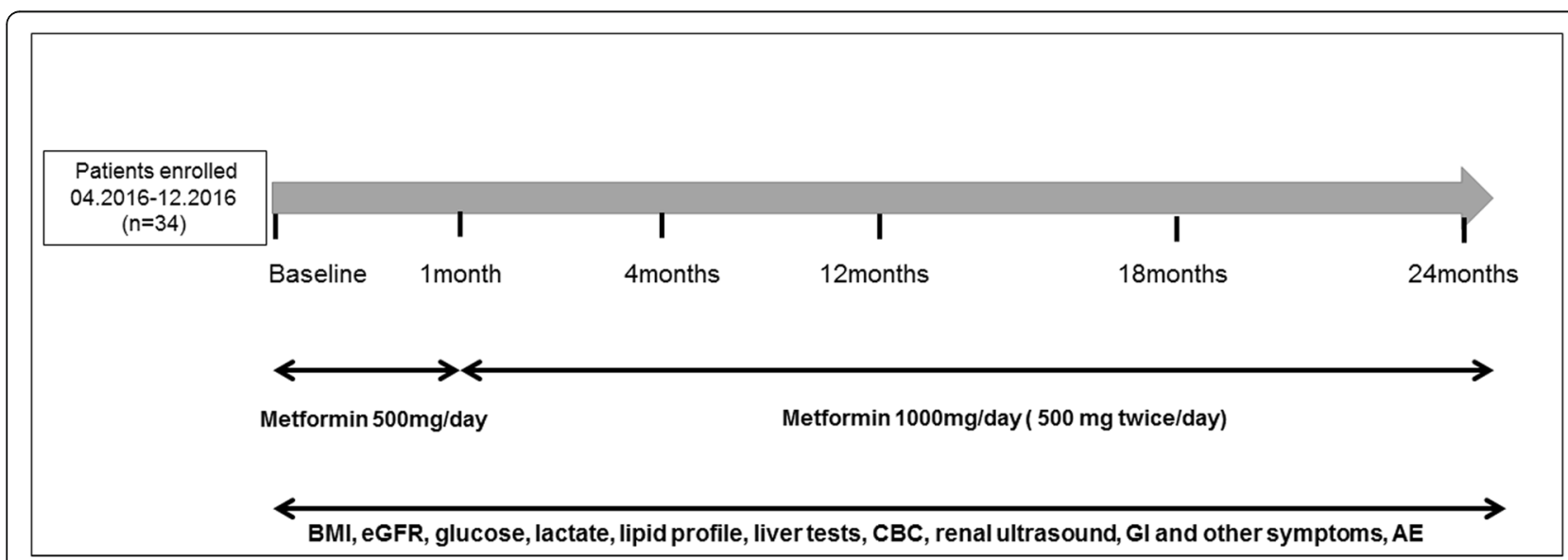

$\mathrm{n}=$ number; $\mathrm{BMI}=$ body mass index; $\mathrm{GFR}=$ estimated glomerular filtration rate; $\mathrm{CBC}=$ complete blood count; $\mathrm{Gl}=$ gastrointestinal; $\mathrm{AE}=$ adverse events;

Fig. 2 Study enrollment, follow-up, patient evaluation and medication dose 
non-gastrointestinal related symptoms. To assess the severity of the GI symptoms, we used the Gastrointestinal Symptoms Rating Scale which consisted of 15 items, each rated on a 7-point Likert scale from no discomfort to very severe discomfort, conducted in an interview manner by a single physician [15]. For the safety analysis, hypoglycemia was defined as blood glucose $<70$ $\mathrm{mg} / \mathrm{dl}$ with or without typical adrenergic symptoms and lactic acidosis was defined as lactic acid level $>4 \mathrm{mmol} / \mathrm{L}$ obtained from venous samples. Mortality was defined as the number of patients who died during the study follow-up. Other adverse events were reported as any adverse event that occurred during the treatment with Metformin. Metformin was permanently discontinued in case of severe gastrointestinal and non-gastrointestinal symptoms, symptomatic hypoglycemia and lactic acidosis. In case of mild/moderate persistent gastrointestinal and non-gastrointestinal symptoms, the dose was decreased until tolerance was obtained. Renal function was evaluated based on serum creatinine, estimated by CKDEPI formula and expressed as estimated glomerular filtration rate (eGFR). BMI was calculated based on kilograms divided by the square of height in meters $\left(\mathrm{kg} / \mathrm{m}^{2}\right)$. A BMI between 25 and $29.9 \mathrm{~kg} / \mathrm{m}^{2}$ falls within the overweight range and $>30 \mathrm{~kg} / \mathrm{m}^{2}$ in the obese range.

\section{Statistical analysis}

Variables were summarized using descriptive statistics as percentages for binary variables, mean \pm standard deviation for continuous normally distributed and median with interquartile range for continuous abnormally distributed variables. Change in eGFR and BMI was expressed as mean difference with the corresponding 95\% confidence intervals and as a percentage. For the primary endpoint, we included all 34 enrolled patients. To assess the secondary endpoint, intention-to-treat (ITT) and per-protocol (PP) analysis was performed. ITT population included all enrolled patients (34 patients), regardless of treatment discontinuation or protocol violation. PP population (16 patients) included only those patients who adhered to protocol. $P$ value $<0.05$ was considered statistically significant. Statistical analysis was performed using IBM SPSS 20 Software (Chicago, Illinois).

\section{Results}

\section{Patients}

A total of 34 patients with ADPKD were enrolled in the study. Sixteen (47\%) out of 34 patients completed the follow-up period at 24 months. Of those who did not adhere to the protocol, 12 (66.7\%) were lost to follow-up, 1 patient $(5.6 \%)$ stopped the medication by his own, in 2 patients $(11.1 \%)$ medication was withdrawn because of adverse events and 3 patients (16.6\%) needed renal replacement therapy (Fig. 1).

\section{Baseline characteristics}

Among the 34 patients, mean age at baseline was $47.29 \pm 11.29$ years, the majority of patients were females (64.7\%) and the mean age at diagnosis was $35.97 \pm 11.66$ years. Only 6 patients out of 34 did not have a family history of ADPKD. Mean BMI was $27.10 \pm 3.65 \mathrm{~kg} / \mathrm{m}^{2}$, $50 \%$ of patients were overweight and $23.5 \%$ were obese, $76.5 \%$ were hypertensive, $67.6 \%$ were dyslipidaemic and $29.4 \%$ were current smokers. Mean eGFR at baseline was $63.75 \pm 34.71 \mathrm{ml} / \mathrm{min} / 1.73 \mathrm{~m}^{2}$ and $67.6 \%$ of patients had CKD stages $1-3$. Urinalysis showed that patients were characterized by hypostenuria. Twenty-one subjects $(61.8 \%)$ were under blood-pressure control medication, 18 out of them receiving angiotensin converting enzyme inhibitors or angiotensin II receptor blockers (Table 1).

\section{Safety analysis}

During the 24 months of follow-up, 18 patients developed adverse events, and $63.6 \%$ of the events were gastrointestinal related. The most common gastrointestinal event was nausea, which appeared in 6 patients $(17.6 \%)$. One patient developed both vomiting and diarrhea. Of the non-gastrointestinal related adverse events, we observed renal cyst complications in 4 patients (11.7\%), including one patient with hemorrhage associated with cysts infection that required antibiotic therapy and one with intracystic hemorrhage. Among other nongastrointestinal related events, we could pointed out rash in one patient and dizziness in another patient. Six of the 18 patients that experienced adverse events (33.3\%) developed them in the first month of treatment, at the initial dose of $500 \mathrm{mg}$ per day. Adverse events that occurred after the first month of treatment, when the dose was increased at $1000 \mathrm{mg}$ per day were: nausea (4 patients), 2 cases of abdominal pain (2 patients), bloating (2 patients) and renal cyst complications (4 patients). Nausea and dizziness, which appeared within the first month of follow-up, were mild and temporary and did not limit the increase of Metformin dose. During followup, no adverse event has determined the decrease of dose, except for the cases where permanently discontinuation was necessary. In our study population, we did not observe any case of hypoglycemia or lactic acidosis and no patient died during the follow-up period (Table 2).

Metformin was permanently discontinued in 2 patients (5.8\%) due to adverse events. In one patient, the reason for discontinuation was severe abdominal pain, which appeared after 4 months of follow-up, at the dosage of $1000 \mathrm{mg} /$ day. Regarding the other patient, we stopped 
Table 1 Baseline characteristics of study population

\begin{tabular}{|c|c|}
\hline Variables & Patients number $(N=34)$ \\
\hline Age (mean, years) & $47.29 \pm 11.29$ \\
\hline \multicolumn{2}{|l|}{ Gender (\%) } \\
\hline Male & $12(35.3 \%)$ \\
\hline Female & $22(64.7 \%)$ \\
\hline Family history of ADPKD (\%) & $28(82.4 \%)$ \\
\hline Mother related & $11(32.4 \%)$ \\
\hline Father related & $17(50 \%)$ \\
\hline None & $6(17.6 \%)$ \\
\hline Age at diagnosis (mean, years) & $35.97 \pm 11.66$ \\
\hline BMI (mean, kg/m²) & $27.10 \pm 3.65$ \\
\hline $25-29.9(\%)$ & 17 (50\%) \\
\hline$\geq 30(\%)$ & $8(23.5 \%)$ \\
\hline \multicolumn{2}{|l|}{ Smoking status (\%) } \\
\hline Current smoker & $10(29.4 \%)$ \\
\hline Past smoker & $6(17.6 \%)$ \\
\hline Non-smoker & $18(52.9 \%)$ \\
\hline History of hematuria (\%) & $4(11.8 \%)$ \\
\hline History of kidney stones (\%) & $12(35.3 \%)$ \\
\hline History of lumbar pain (\%) & $28(82.4 \%)$ \\
\hline History of UTI (\%) & $14(44.1 \%)$ \\
\hline HTN (\%) & $26(76.5 \%)$ \\
\hline Dyslipidemia (\%) & $23(67.6 \%)$ \\
\hline SBP (mean, mmHg) & $136.18 \pm 15.42$ \\
\hline DBP (mean, mmHg) & $83.09 \pm 11.54$ \\
\hline Serum creatinine (median, mg/dl) & $1.11(0.88-2.61)$ \\
\hline eGFR (mean, $\mathrm{ml} / \mathrm{min} / 1.73 \mathrm{~m}^{2}$ ) & $60.45 \pm 34.71$ \\
\hline \multicolumn{2}{|l|}{ CKD stage (\%) } \\
\hline 1 & $8(23.5 \%)$ \\
\hline 2 & $10(29.4 \%)$ \\
\hline 3 & $5(14.7 \%)$ \\
\hline 4 & $6(17.6 \%)$ \\
\hline 5 & $5(14.7 \%)$ \\
\hline Serum urea (median, mg/dl) & $59(36.42-80.75)$ \\
\hline Glucose level (mean, mg/dl) & $87.75 \pm 15.90$ \\
\hline Total cholesterol (mean, mg/dl) & $186.88 \pm 37.32$ \\
\hline Triglycerides (median, mg/dl) & $95.50(70-145.25)$ \\
\hline Antihypertensive treatment (\%) & $21(61.8 \%)$ \\
\hline ACEI/ARB (\%) & $18(52.9 \%)$ \\
\hline Urine specific gravity (mean) & $1012 \pm 6.39$ \\
\hline
\end{tabular}

$N$ number, $A D P K D$ autosomal dominant polycystic kidney disease, $B M I$ body mass index, UTI urinary tract infections, HTN arterial hypertension, SBP systolic blood pressure, DBP diastolic blood pressure, GFR glomerular filtration rate, $C K D$ chronic kidney disease, $A C E l$ angiotensin converting enzyme inhibitors, $A R B$ angiotensin II receptor blockers
Table 2 Adverse events during the study period

\begin{tabular}{ll}
\hline Adverse event type & No. of patients with event (\%) \\
\hline Gastrointestinal related & $6(17.6 \%)$ \\
Nausea & $1(2.9 \%)$ \\
Vomiting & $1(2.9 \%)$ \\
Diarrhea & $2(5.8 \%)$ \\
Abdominal pain & $2(5.8 \%)$ \\
Bloating & \\
Non-gastrointestinal related & $1(2.9 \%)$ \\
Dizziness & $1(2.9 \%)$ \\
Rash & $4(11.7 \%)$ \\
Renal cyst complications & \\
(infection and/or hemorrhage) & $0(0 \%)$ \\
Lactic acidosis & $0(0 \%)$ \\
Hypoglycemia & $0(0 \%)$ \\
Death &
\end{tabular}

Metformin because of severe gastrointestinal and skin manifestations, including vomiting, diarrhea and rash, which appeared during the second week, at the initial dose of $500 \mathrm{mg}$ per day.

\section{Secondary endpoints analysis \\ Change in estimated glomerular filtration rate over 24 months of treatment}

Evolution of renal function in ITT population is shown in Table 3a. The observed change of mean eGFR after 4, 12 and 24 months of treatment was of $+1.71 \mathrm{ml} / \mathrm{min} / 1.73 \mathrm{~m}^{2}$ (95\% CI: -16.56 to $19.98 ; P=$ 0.85 ), $-1.03 \mathrm{ml} / \mathrm{min} / 1.73 \mathrm{~m}^{2}$ (95\% CI: -21.28 to 19.22 , $P=0.91)$ and $-1.57 \mathrm{ml} / \mathrm{min} / 1.73 \mathrm{~m}^{2}$ (95\% CI: -22.28 to $19.14, P=0.87$ ), respectively.

Evolution of renal function in per-protocol population is shown in Table 3b. The change from baseline of mean eGFR was of $+1.81 \mathrm{ml} / \mathrm{min} / 1.73 \mathrm{~m}^{2}$ (95\% CI: -21.60 to $24.68, \mathrm{P}=0.87$ ) after the first 4 months, followed by $-0.65 \mathrm{ml} / \mathrm{min} / 1.73 \mathrm{~m}^{2}$ (95\% CI: -24.94 to 23.64, $P=0.95)$ at 12 months and $-4.57 \mathrm{ml} / \mathrm{min} /$ $1.73 \mathrm{~m}^{2}$ (95\% CI: -28.03 to $18.89, P=0.69$ ) at 24 months of treatment.

Rate changes of eGFR from baseline, corresponding to those of mean eGFR, were illustrated in Fig. 3a and b. After 24 months of treatment with Metformin, according to ITT analysis, eGFR had changed by $-2.59 \%$ and in agreement with PP analysis, by $-7.2 \%$.

Change in body mass index over 24 months of treatment Evolution of BMI is shown in Table 3a and b. At 24 months, mean BMI change from baseline was $-1.10 \mathrm{~kg} / \mathrm{m}^{2}$ (95\% CI:-3.22 to $1.02, P=0.30$ ) in ITT population and $-0.80 \mathrm{~kg} / \mathrm{m}^{2}(95 \% \mathrm{CI}:-3.27$ to $1.67, P=0.51)$ in patients included in PP analysis. The rate changes of 
Table 3 Evolution of eGFR and BMI over 24 months of treatment in intention-to-treat and per-protocol population

\begin{tabular}{|c|c|c|c|c|c|c|c|c|c|}
\hline No. of patients & Study period & $\begin{array}{l}\text { Mean eGFR value } \\
\left(\mathrm{ml} / \mathrm{min} / 1.73 \mathrm{~m}^{2}\right)\end{array}$ & $\begin{array}{l}\text { Mean eGFR } \\
\text { change } \\
\left(\mathrm{ml} / \mathrm{min} / 1.73 \mathrm{~m}^{2}\right)\end{array}$ & $95 \% \mathrm{Cl}$ & $P$ Value & $\begin{array}{l}\text { Mean BMl } \\
\text { Value } \\
\left(\mathrm{kg} / \mathrm{m}^{2}\right)\end{array}$ & $\begin{array}{l}\text { Mean BMI } \\
\text { change } \\
\left(\mathrm{kg} / \mathrm{m}^{2}\right)\end{array}$ & $95 \% \mathrm{Cl}$ & $P$ Val \\
\hline \multicolumn{10}{|c|}{ A. Mean eGFR and BMI changes in intention to treat population } \\
\hline 34 & Baseline & $60.45 \pm 34.71$ & - & - & - & $27.10 \pm 3.65$ & - & - & - \\
\hline 28 & 1 month & $61.41 \pm 34.17$ & 0.96 & -16.63 to -18.55 & 0.91 & $26.94 \pm 3.58$ & -0.16 & -2.00 to 1.68 & 0.86 \\
\hline 25 & 4 months & $62.16 \pm 34.53$ & 1.71 & -16.56 to 19.98 & 0.85 & $26.45 \pm 3.48$ & -0.65 & -2.53 to 1.23 & 0.49 \\
\hline 20 & 12 months & $59.42 \pm 37.67$ & -1.03 & -21.28 to 19.22 & 0.91 & $26.27 \pm 3.32$ & -0.80 & -2.82 to 1.16 & 0.40 \\
\hline 16 & 24 months & $58.88 \pm 32.32$ & -1.57 & -22.28 to 19.14 & 0.87 & $26.00 \pm 3.07$ & -1.10 & -3.22 to 1.02 & 0.30 \\
\hline \multicolumn{10}{|c|}{ B. Mean eGFR and BMI changes in per protocol population } \\
\hline 16 & Baseline & $63.45 \pm 32.68$ & - & - & - & $26.80 \pm 3.76$ & - & - & - \\
\hline 16 & 1 month & $64.83 \pm 31.68$ & 1.38 & -21.85 to 24.61 & 0.90 & $26.56 \pm 3.82$ & -0.24 & -2.97 to 2.49 & 0.85 \\
\hline 16 & 4 months & $65.26 \pm 30.65$ & 1.81 & -21.60 to 24.68 & 0.87 & $26.34 \pm 3.60$ & -0.46 & -3.11 to 2.19 & 0.76 \\
\hline 16 & 12 months & $62.80 \pm 34.58$ & -0.65 & -24.94 to 23.64 & 0.95 & $26.31 \pm 3.46$ & -0.49 & -3.09 to 2.11 & 0.70 \\
\hline 16 & 24 months & $58.88 \pm 32.32$ & -4.57 & -28.03 to 18.89 & 0.69 & $26.00 \pm 3.07$ & -0.80 & -3.27 to 1.67 & 0.51 \\
\hline
\end{tabular}

No. number, $\mathrm{Cl}$ confidence interval, eGFR estimated glomerular filtration rate, $B M I$ body mass index

BMI from baseline value, concurrent with those of mean BMI, were of $-4.05 \%$ and $-2.98 \%$, respectively (Fig. 4a and b).

\section{Renal replacement therapy end-point}

During the study period, 3 patients (8.8\%) out of 34 needed renal replacement therapy. Of those, 2 became hemodialysis dependent and 1 patient underwent a preemptive living donor kidney transplantation. All patients were female, two had CKD stage 5 at the beginning of the study and received a dose of $500 \mathrm{mg} /$ day of Metformin and the patient who underwent transplantation had CKD stage 4 . None of these patients had a precipitating factor or an acute event before the RRT was started. The time from Metformin starting to RRT initiation was of 4 and 12 months for the two patients on hemodialysis and 12 months for the patient that underwent kidney transplantation.

\section{Discussion}

Results from our study showed that treatment with Metformin was well tolerated in all stages of CKD, with few adverse events, and it was not associated with any changes in either eGFR or BMI at any time point. To our knowledge, this is the first report from a prospective, interventional study about Metformin in patients with ADPKD.

The most common adverse event found in our study was nausea (17.6\%), but this manifestation was mild and transitory, no dose reduction or permanently discontinuation was required. This finding is in agreement with results from other studies, where nausea was among the most common adverse events in diabetic and non-diabetic patients treated with Metformin [16, 17]. It has been shown that the frequency of nausea was of $10 \%$ and that treatment discontinuation rate due to nausea was $3 \%$ in patients with type 2 diabetes treated with $1 \mathrm{~g} /$ day of Metformin [16]. Findings from our study do not exceed the current evidences regarding cyst infection in ADPKD patients and are in line with those of Sallée $M$. et al., who showed that $8.6 \%$ of patients developed cyst infection [18]. Hypoglycemia is a rare event in patients treated with Metformin, but the risk of hypoglycemia increases in those with CKD $[19,20]$. In our study population, hypoglycemia was absent. The use of Metformin in patients with CKD is limited by guideline contraindications because of the fear for lactic acidosis. The European Medicines Agency and Food and Drug Association recommended to use Metformin up to an eGFR of $30 \mathrm{ml} / \mathrm{min} / 1.73 \mathrm{~m}^{2}$, but this cut-off value was not based on results from prospective studies of pharmacodynamics and pharmacokinetics [21, 22]. The incidence of Metformin-associated lactic acidosis was 4.3 per 100,000 patient-years in a 2010 Cochrane analysis [23]. In this systematic review, Salpeter et al. demonstrated that Metformin was not associated with an increased risk of lactic acidosis compared to other antidiabetic drugs. Moreover, 53\% of the studies included patients with CKD, including 37,360 patient-years with no events of lactic acidosis [23]. This data is supported by an observational cohort study from the Swedish National Diabetes Register, which analyzed 51,675 patients with type 2 diabetes mellitus, including $12.3 \%$ with eGFR $\leq 60$ $\mathrm{ml} / \mathrm{min} / 1.73 \mathrm{~m}^{2}$ and has shown that lactic acidosis is a rare event even in those with eGFR between 30 and $45 \mathrm{ml} /$ $\mathrm{min} / 1.73 \mathrm{~m}^{2}$ [24]. Duong et al. reported no adverse events or a relationship between Metformin, lactic acid levels or lactic acidosis in 7 patients with advanced CKD, including 2 patients on dialysis, who were treated with lower doses 


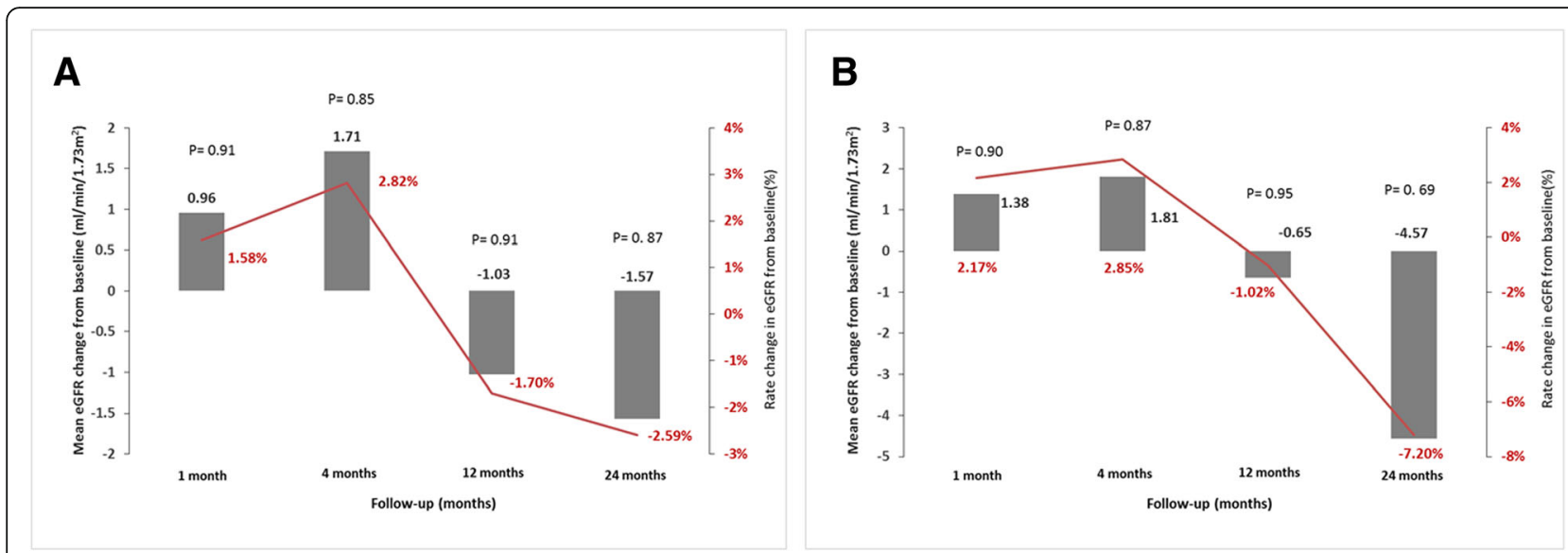

*eGFR- estimated glomerular filtration rate

Fig. 3 Evolution of eGFR over 24 months of treatment in intention-to-treat (a) and per-protocol analysis (b)

of Metformin, between 250 and $500 \mathrm{mg} /$ day [25]. Of note, a recent study by Lalau et al. showed that adjusting the dose of Metformin in patients with moderate-severe CKD seems to be safe and remained pharmacologically efficient. A dose of $500 \mathrm{mg} /$ day does not determine a drug concentration $>5 \mathrm{mg} / \mathrm{L}$, considered the safe upper limit, not even in CKD stages 4-5 and a dose of $1000 \mathrm{mg} /$ day produced a drug concentration $>5 \mathrm{mg} / \mathrm{L}$ in 1 patient from CKD stages 4-5. Lactate concentration did not exceed $5 \mathrm{mmol} / \mathrm{L}$ in any patient [26]. In accordance with these findings, our study included patients in all stages of CKD and no case of lactic acidosis was reported.

Analysis of the kidney function showed no change in kidney function after 24 months of treatment, but with an initial increase within the first 4 months. This initial, transitory improvement in eGFR could be associated with the weight loss and subsequent change in BMI. It is well known that Metformin produces weight loss and
BMI reduction even in overweight or obese patients without diabetes [13]. Moreover, one study has shown that weight loss leads to kidney function improvement, in those with baseline eGFR $<60 \mathrm{ml} / \mathrm{min} / 1.73 \mathrm{~m}^{2}$, especially in the first 6 months after dietary restriction [27]. In our study, the greater rate in BMI change occurred in the first 4 months $(-2.39 \%)$ and we also observed a concomitant variation in median serum creatinine (from 1.11 to $1.03 \mathrm{mg} / \mathrm{dl}$ ) that could be influenced by muscle mass loss, thus affecting the eGFR value in this early period (mean eGFR change from baseline: $+1.71 \mathrm{ml} / \mathrm{min} /$ $1.73 \mathrm{~m}^{2}$ ). Two randomized control trials (NCT02656017; NCT02903511) involving Metformin safety and efficacy in ADPKD are ongoing. One retrospective, case-control study, which selected 7 diabetic ADPKD patients treated with Metformin and 7 matched non-diabetic ADPKD controls, evaluated the effect of Metformin on renal function progression during a 3 year follow-up. An overall
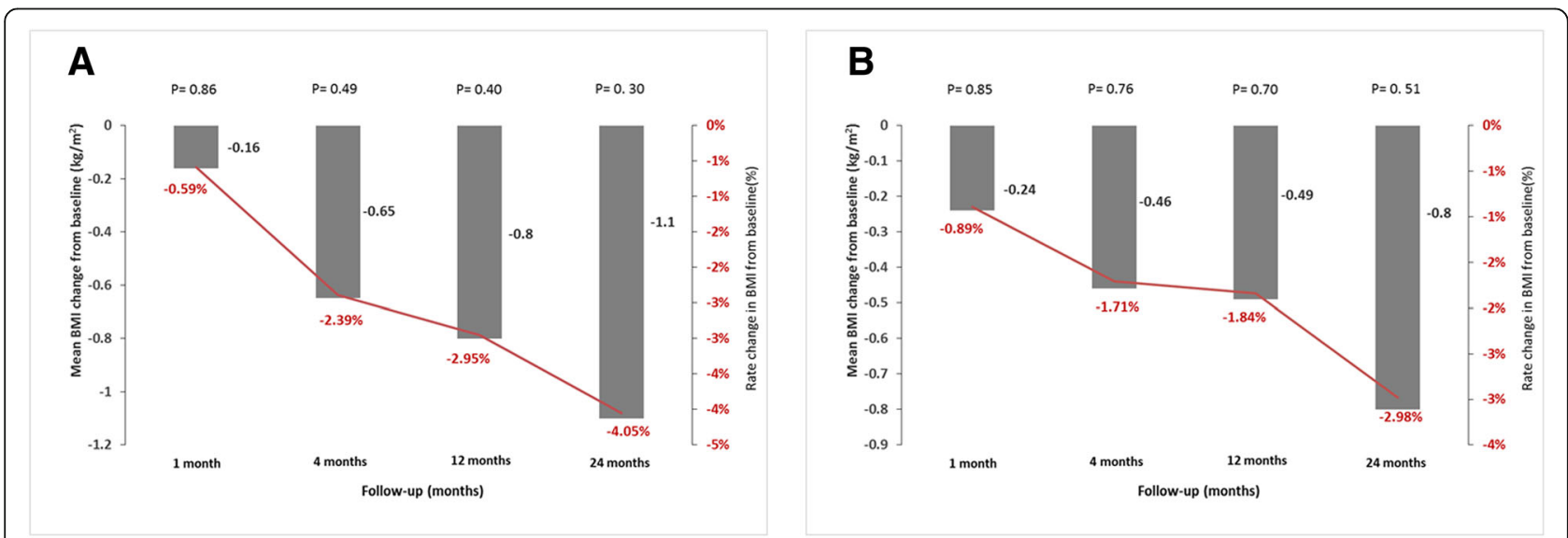

*BMI- body mass index

Fig. 4 Evolution of BMI over 24 months of treatment in intention-to-treat (a) and per-protocol analysis (b) 
crude loss of eGFR of $-0.9 \mathrm{ml} / \mathrm{min} / 1.73 \mathrm{~m}^{2}$ estimated by a linear mixed model at 36 months was reported in the Metformin group [28]. Our ITT analysis was in line with these results, as we found a change in mean eGFR of $-1.57 \mathrm{ml} /$ $\mathrm{min} / 1.73 \mathrm{~m}^{2}$ after 24 months of treatment. If we refer to other clinical trials testing medication to slow eGFR decline in ADPKD, in the TEMPO 3:4 study, which included patients with eGFR $>60 \mathrm{ml} / \mathrm{min} / 1.73 \mathrm{~m}^{2}$, Tolvaptan slowed the decline in kidney function after 36 months $(-2.61$ vs $-3.81[\mathrm{mg} / \mathrm{ml}]^{-1}$ per year), but was associated with an increased rate of withdrawal due to adverse events [29]. REPRISE trial, which included patients with CKD stages $2-4$, showed a mean eGFR change from baseline of -2.34 $\mathrm{ml} / \mathrm{min} / 1.73 \mathrm{~m}^{2}$ in the Tolvaptan group, as compared with $-3.61 \mathrm{ml} / \mathrm{min} / 1.73 \mathrm{~m}^{2}$ in the placebo group after 12 months (difference, $1.27 \mathrm{ml} /$ minute $/ 1.73 \mathrm{~m}^{2}$; $95 \% \mathrm{CI}$, 0.86 to $1.68 ; P<0.001$ ) [30]. The eGFR decline in these studies was higher than what we found at 12 and 24 months in ITT as well as in PP analysis. The efficacy of Sirolimus, an mTOR inhibitor, that partially shares some mechanisms of action with Metformin in cystogenesis inhibition, was tested in clinical trials. Despite the fact that SUISSE trial and the study of Braun et al. showed a favorable GFR evolution from baseline of $+0.2 \mathrm{ml} / \mathrm{min} / 1.73 \mathrm{~m}^{2}$ after 12 months $(P=0.07)$ and $+1.6 \mathrm{ml} / \mathrm{min} / 1.73 \mathrm{~m}^{2}$ after 18 months $(\mathrm{P}<0.001)$ in the Sirolimus group, a meta-analysis demonstrated that mTORC1 inhibitors had no significant effect on eGFR change in patients with ADPKD $(P=0.22)$ [31-33].

In our study population, $73.5 \%$ were obese or overweight at baseline and we found a progressive decline in BMI, more accelerated within the first 4 months. Nowak et al. proved that obesity and overweight patients with ADPKD have a greater decline in eGFR that normal weight ones [12]. Obesity and ADPKD seem to share some common pathways. Positive energy balance, overnutrition and obesity can produce a hyperactivity of mTOR pathway via PI3k/Akt activation, may activate mTORC1/S6K and reduce AMPK activity. Hyperactivation of mTORC1/S6K pathway and decreased activity of AMPK are important players in ADPKD cystogenesis, regarding proliferation and cyst fluid secretion [34, 35]. Based on this, the use of Metformin in ADPKD patients could decrease energy intake, stimulate AMPK activation and produce mTOR pathway inhibition, which promotes inhibition of cystogenesis and weight gain, the latter having in turn a favorable effect on disease progression and kidney function. In our patients, the slower decline in eGFR compared to other studies could be explained by the concomitant weight loss.

Our study has several limitations. The absence of a control group cannot distinguish between the effect of the treatment and the natural evolution of the disease. The reason that we chose a single-arm design was that we mainly wanted to point out preliminary results regarding the safety and tolerability of Metformin in ADPKD patients. Other limitations are the small sample size and the single-center study enrollment. Another important limitation of the study is the high rate of dropout (54\%), which requires cautious interpretation of tolerability and safety results. Regarding the 12 patients that were lost to follow-up, we do not have any information whether their decision to drop-out was based on medication intolerance or adverse reactions or not, which may lead to an under-estimation of tolerability. The strengths of our study were the prospective design, the intention-to-treat analysis, the length of the followup period and the inclusion of patients with advanced CKD stages. Given the small sample size, absence of a control arm and high rate of drop-out we consider that our findings need to be evaluated in a large randomized controlled trial.

\section{Conclusion}

In conclusion, the results from this single-arm pilot study showed that Metformin was well tolerated, with a good safety profile in patients with ADPKD, even in those with advanced CKD and it was not associated with change in eGFR or BMI across the 24 months of followup.

\section{Abbreviations \\ ADPKD: Autosomal dominant polycystic kidney disease; AMPK: Adenosine monophosphate-activated protein kinase; BMl: Body mass index; \\ CAMP: Cyclic adenosine monophosphate; CFTR: Chloride channel cystic fibrosis transmembrane conductance regulator; CKD: Chronic kidney disease; eGFR: Estimated glomerular filtration rate; ERA-EDTA: European Renal Association-European Dialysis and Transplant Association; ITT: Intention to treat; JAK/STAT: Just another kinase/ signal transducer and activator of transcription; MAPK/ERK: Mitogen-activated protein kinase/extracellular signal-regulated kinase; PP: Per protocol; RRT: Renal replacement therapy; TSC2: Protein of tuberous sclerosis 2}

\section{Acknowledgments}

We are thankful to Dr. Madalina Sorohan for providing language help, to all doctors and nurses from the nephrology department who contributed to this study and we also thank the participants of the study.

\section{Authors' contributions}

The authors of the manuscript contributed to this article in the following way: BMS,GI,IS,CB designed the study protocol, patient informed consent and wrote the main text; $B M S, A A, R J, B O, G M$ collected the data, prepared tables and figures; BMS analyzed and interpreted the data;

$B M S, G I, A A, B O, R J, G M, I S, C B$ critically revised the manuscript. All of the authors read and approved the final version of the manuscript.

\section{Funding}

This research did not receive any specific grant from funding agencies in the public, commercial, or not-for-profit sectors.

\section{Availability of data and materials}

The datasets used and/or analysed during the current study are available from the corresponding author on reasonable request.

\section{Ethics approval and consent to participate}

This study was approved by the local ethics committee of Fundeni Clinical Institute (No.39436) and all patients signed the informed consent. 


\section{Consent for publication}

Not applicable.

\section{Competing interests}

All the authors declared that they have no competing interests.

\section{Author details}

'Carol Davila University of Medicine and Pharmacy, Bucharest, Romania. ${ }^{2}$ Nephrology Department, Fundeni Clinical Institute, Fundeni Street No. 258, ZIP Code 022328, District No.2, Bucharest, Romania. ${ }^{3}$ Center of Uronephrology and Renal Transplantation, Fundeni Clinical Institute, Bucharest, Romania.

\section{Received: 5 March 2019 Accepted: 11 July 2019}

\section{Published online: 23 July 2019}

\section{References}

1. Torres VE, Harris PC, Pirson Y. Autosomal dominant polycystic kidney disease. Lancet. 2007;369(9569):1287-301.

2. Solazzo A, Testa F, Giovanella S, et al. The prevalence of autosomal dominant polycystic kidney disease (ADPKD): a meta-analysis of European literature and prevalence evaluation in the Italian province of Modena suggest that ADPKD is a rare and underdiagnosed condition. PLoS One. 2018;13(1):e0190430

3. Willey CJ, Blais JD, Hall AK, et al. Prevalence of autosomal dominant polycystic kidney disease in the European Union. Nephrol Dial Transplant. 2017;32(8):1356-63.

4. Iliuta IA, Kalatharan V, Wang K, et al. Polycystic kidney disease without an apparent family history. J Am Soc Nephrol. 2017;28(9):2768-76.

5. Cornec-Le Gall E, et al. Genkyst study group; HALT progression of polycystic kidney disease group; consortium for radiologic imaging studies of polycystic kidney disease, Harris PC. Monoallelic mutations to DNAJB11 cause atypical autosomal-dominant polycystic kidney disease. Am J Hum Genet. 2018;102(5):832-44.

6. Ong AC, Harris PC. Molecular pathogenesis of ADPKD: the polycystin complex gets complex. Kidney Int. 2005;67(4):1234-47.

7. Malekshahabi T, Khoshdel Rad N, Serra AL, et al. Autosomal dominant polycystic kidney disease: disrupted pathways and potential therapeutic interventions. J Cell Physiol. 2019;15.

8. Lanktree MB, Chapman AB. New treatment paradigms for ADPKD: moving towards precision medicine. Nat Rev Nephrol. 2017;13(12):750-68.

9. Takiar V, Nishio S, Seo-Mayer P, et al. Activating AMP-activated protein kinase (AMPK)slows renal cystogenesis. Proc Natl Acad Sci U S A. 2011; 108(6):2462-7.

10. Chang MY, Ma TL, Hung CC, et al. Metformin Inhibits Cyst Formation in a Zebrafish Model of Polycystin-2 Deficiency. Sci Rep. 2017;7(1):7161. Published 2017 Aug 2.

11. Schrier RW, Brosnahan G, Cadnapaphornchai MA, et al. Predictors of autosomal dominant polycystic kidney disease progression. J Am Soc Nephrol. 2014;25(11):2399-418.

12. Nowak KL, You Z, Gitomer B, et al. Overweight and obesity are predictors of progression in early autosomal dominant polycystic kidney disease. J Am Soc Nephrol. 2018;29(2):571-8.

13. Hui F, Zhang $Y$, Ren $T$, et al. Role of metformin in overweight and obese people without diabetes: a systematic review and network meta-analysis. Eur J Clin Pharmacol. 2018;3.

14. Pei $Y$, et al. Unified criteria for ultrasonographic diagnosis of ADPKD. J Am Soc Nephrol. 2009;20(1):205-12.

15. Revicki DA, Wood M, Wiklund I, et al. Reliability and validity of the gastrointestinal symptom rating scale in patients with gastroesophageal reflux disease. Qual Life Res. 1998;7:75-83.

16. Garber AJ, Duncan TG, Goodman AM, et al. Efficacy of metformin in type II diabetes: results of a double-blind, placebo-controlled, dose-response trial. Am J Med. 1997:103:491-7.

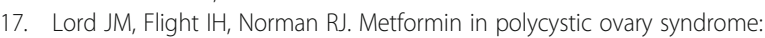
systematic review and meta-analysis. BMJ. 2003;327(7421):951-3.

18. Sallée M, Rafat C, Zahar JR, et al. Cyst infections in patients with autosomal dominant polycystic kidney disease. Clin J Am Soc Nephrol. 2009;4(7):1183-9.

19. Bolen S, Feldman L, Vassy J et al. Systematic review: comparative effectiveness and safety of oral medications for type 2 diabetes mellitus.
Ann Intern Med. 2007 Sep 18;147(6):386-99. Epub 2007 Jul 16. Review. Erratum in: Ann Intern Med. 2007 Dec 18;147(12):887.

20. Moen MF, Zhan M, Hsu VD, et al. Frequency of hypoglycemia and its significance in chronic kidney disease. Clin J Am Soc Nephrol. 2009;4(6): 1121-7.

21. The EuropeanMedicines Agency (EMA). Use of metformin to treat diabetes now expanded to patients with moderately reduced kidney function: recommendations for patients with kidney impairment updated in product information [Internet], 2016.Available from http://www.ema.europa.eu/docs/ en_GB/document_library/Press_release/2016/10/WC500214248.pdf. Accessed 5 Jan 2019.

22. The U.S. Food and Drug Administration (FDA). Drug Safety Communication: FDA revises warnings regarding use of the diabetes medicine metformin in certain patients with reduced kidney function [Internet], 2016. Available from https:// www.fda.gov/downloads/Drugs/DrugSafety/ UCM494140.pdf. Accessed 5 Jan 2019.

23. Salpeter SR, Greyber E, Pasternak GA, et al. Risk of fatal and nonfatal lactic acidosis with metformin use in type 2 diabetes mellitus (review). Cochrane Database Syst Rev. 2010;1:CD002967.

24. Ekström, Nils et al. Effectiveness and safety of metformin in 51675 patients with type 2 diabetes and different levels of renal function: a cohort study from the Swedish National Diabetes Register. BMJ open vol. 2,4 e001076. 13 Jul. 2012.

25. Duong JK, Roberts DM, Furlong TJ, et al. Metformin therapy in patients with chronic kidney disease. Diabetes Obes Metab. 2012;14(10):963-5.

26. Lalau JD, Kajbaf F, Bennis $Y$, et al. Metformin treatment in patients with type 2 diabetes and chronic kidney disease stages 3A, 3B, or 4. Diabetes Care. 2018;41(3):547-53.

27. Tirosh A, et al. Renal function following three distinct weight loss dietary strategies during 2 years of a randomized controlled trial. Diabetes Care. 2013;36(8):2225-32.

28. Pisani A, Riccio E, Bruzzese D, et al. Metformin in autosomal dominant polycystic kidney disease: experimental hypothesis or clinical fact? BMC Nephrol. 2018;19(1):282.

29. Torres VE, Chapman AB, Devuyst O, et al. TEMPO 3:4 trial Investigators. Tolvaptan in patients with autosomal dominant polycystic kidney disease. N Engl J Med. 2012;367(25):2407-18.

30. Torres VE, Chapman AB, Devuyst O, et al. REPRISE trial Investigators. Tolvaptan in later-stage autosomal dominant polycystic kidney disease. N Engl J Med. 2017;377(20):1930-42.

31. Serra AL, Poster $D$, Kistler $A D$, et al. Sirolimus and kidney growth in autosomal dominant polycystic kidney disease. N Engl J Med. 2010;363(9): 820-9.

32. Braun WE, Schold JD, Stephany BR, et al. Low-dose rapamycin(sirolimus) effects in autosomal dominant polycystic kidney disease: an open-label randomized controlled pilot study. Clin J Am Soc Nephrol. 2014;9(5):881-8.

33. Myint TM, Rangan GK, Webster AC. Treatments to slow progression of autosomaldominant polycystic kidney disease: systematic review and metaanalysis ofrandomized trials. Nephrology (Carlton). 2014;19(4):217-26.

34. Jia G, Aroor AR, Martinez-Lemus LA, et al. Overnutrition, mTOR signaling, and cardiovascular diseases. Am J Physiol Regul Integr Comp Physiol. 2014; 307(10):R1198-206.

35. Dann SG, Selvaraj A, Thomas G. mTOR Complex1-S6K1 signaling: at the crossroads of obesity, diabetes and cancer. Trends Mol Med 2007;13(6):252259. Epub 2007 Apr 23. Review.

\section{Publisher's Note}

Springer Nature remains neutral with regard to jurisdictional claims in published maps and institutional affiliations. 